\title{
Entrenamiento para olvidos cotidianos y mejora de la memoria en mayores: resultados de efectividad
}

\author{
María Eugenia Domínguez-Orozco*
}

\footnotetext{
Magíster en Psicogerontología, Universitat de Barcelona. Consultora independiente, Cádiz, España.

Correo electrónico:

medorozco@hotmail.com
}

Recibido: 22 de junio del 2015

Aprobado: 12 de noviembre del 2015

Cómo citar este artículo: Domínguez, M. E. (2015). Entrenamiento para olvidos cotidianos y mejora de la memoria en mayores: resultados de efectividad. Pensando Psicología, 11(18), 73-84. doi: http://dx.doi. org/10.16925/pe.v11i18.1220

\section{Resumen}

Introducción: las quejas subjetivas y los olvidos cotidianos en personas mayores pueden ser modificables mediante el entrenamiento cognitivo y estrategias y mnemotécnicas para mejorar la memoria. Diversos métodos y programas de entrenamiento específicos sobre memoria se han desarrollado en España con resultados exitosos en su implantación. Objetivos: el propósito principal de este artículo ha sido mostrar la efectividad del entrenamiento de diferentes estrategias y técnicas en el rendimiento del recuerdo en mayores. Concretamente, se realizaron ensayos con la técnica de asociación para caras y nombres, las estrategias de visualización y categorización, así como el método loci; todas experimentadas con los mayores participantes en un taller de mejora de la memoria. Resultados y conclusiones: el diseño del entrenamiento de carácter multifactorial modular y la inclusión de estos procedimientos mnemotécnicos facilitó la ganancia en el recuerdo. Los análisis de resultados pusieron de manifiesto el incremento significativo al entrenar tareas de memoria prospectiva y retrospectiva, tales como "acciones a realizar en una mañana" a través del método loci, la mejora de la codificación de la información a través de la categorización, la optimización de la memoria sensorial visual con la visualización y, por último, mejora de los déficits asociativos en memoria episódica en el recuerdo de nombres de personas.

Palabras clave: asociación y categorización, entrenamiento de la memoria, método loci, olvidos cotidianos, visualización. 


\title{
Training for People Who Are Forgetful in Daily Life and to Improve Memory in Older People: Results of Effectiveness
}

\begin{abstract}
Introduction: Subjective complaints and forgetfulness in daily life among older people can be modified through cognitive training and mnemonic strategies and techniques to improve memory. Diverse methods and specific memory training programs have been developed in Spain with successful results from their implantation. Objectives: The main purpose of this article is to show the effectiveness of the training using different strategies and techniques for improving memory performance among older people. Specifically, trials were carried out with the association technique using faces and names, visualization and categorization strategies along with the loci method, all with older participants during a memory improvement workshop. Results and conclusions: The modular multifactorial design of the training and inclusion of these mnemonic procedures facilitated memory improvement. The analysis of the results showed a significant increase by means of training using prospective and retrospective tasks, such as "actions to be carried out in the morning", and using the loci method, improvement in information codification through categorization, visual sensorial memory optimization using visualization, and finally, improvement of associative deficits in episodic memory in recalling people's names.
\end{abstract}

Keywords: association and categorization, memory training, loci method, daily forgetfulness, visualization.

\section{Treinamento para esquecimentos cotidianos e melhora da memória em idosos: resultados de efetividade}

\section{Resumo}

Introdução: as reclamações subjetivas e os esquecimentos cotidianos em pessoas idosas podem ser modificáveis mediante o treinamento cognitivo e estratégias e mnemotécnicas para melhorar a memória. Diversos métodos e programas de treinamento específicos sobre memória vêm sendo desenvolvidos na Espanha com resultados bem-sucedidos em sua implantação. Objetivos: o propósito principal deste artigo é mostrar a efetividade do treinamento de diferentes estratégias e técnicas no rendimento da recordação em idosos. Concretamente, realizaram-se ensaios com a técnica de associação para caras e nomes, as estratégias de visualização e categorização, bem como o método loci; todas experimentadas com os idosos participantes de uma oficina de aperfeiçoamento da memória. Resultados e conclusões: o desenho do treinamento de caráter multifatorial modular e a inclusão desses procedimentos mnemotécnicos facilitou o ganho na recordação. As análises de resultados manifestaram o aumento significativo na pontuação ao treinar tarefas de memória prospectiva e retrospectiva, tais como "ações a realizar numa manhã" por meio do método loci, a melhora da codificação da informação mediante a categorização, a otimização da memória sensorial visual com a visualização e, por último, a melhora dos déficits associativos em memória episódica na recordação de nomes de pessoas.

Palavras-chave: associação e categorização, treinamento da memória, método loci, esquecimentos cotidianos, visualização. 


\section{Introducción}

Con el paso de los años nuestro sistema cognitivo puede sufrir modificaciones en su funcionamiento. La capacidad de adaptación de estructuras neuronales al redirigir procesos se origina a través de la plasticidad cerebral (Stern, 2002). La reserva cognitiva en la vejez compensa y/o previene el declive mediante el uso más eficiente de los mecanismos cerebrales (Calero, 2000), convirtiéndose en el fundamento que permite a la cognición ser entrenable (Stern, 2013; Calero-García y Navarro-González, 2006). Esto en razón, además, a la capacidad de aprendizaje en los mayores.

Entre los procesos cognitivos más estudiados, la memoria ha recibido mayor auge. Los fallos y quejas de memoria se han convertido en uno de los primeros motivos de consulta de salud en atención especializada (Montenegro et al., 2013), y de ahí su relevancia en la intervención. Como apuntaron Pousada y De la Fuente (2006), el interés en la investigación específica de los cambios en la memoria asociados al envejecimiento parten de las siguientes orientaciones: a) explicaciones sobre el papel de la metamemoria; b) la basada en los niveles de procesamiento; c) la centrada en la fase de recuperación de la información; y d) la referente a los cambios en recursos de procesamiento. Las estrategias y técnicas mnemotécnicas que guían las intervenciones están basadas en estas orientaciones. A continuación se explicará qué acontece en las diferentes memorias en los mayores.

\section{La memoria: quejas subjetivas y olvidos cotidianos}

Las personas mayores suelen buscar el mejoramiento de su memoria de eventos cotidianos específicos, tales como el recuerdo de nombres de personas, fechas importantes, números de teléfono, pérdida de objetos del hogar, cosas de la compra, olvidar tomar la medicación, etc. Estos eventos hacen referencia a las quejas subjetivas de memoria, las cuales tienen una alta frecuencia durante el envejecimiento y son cuestiones imprescindibles para mantener la autonomía (Cortés y Estrada, 2000; McDougall, 2000). Esto es lo que Montejo y Montenegro (2006) denominan memoria cotidiana, la cual comprende: a) olvidos cotidianos retrospectivos (recordar cosas hechas o aprendidas, acciones automáticas, recuerdo de nombres); b) prospectivos (recordar cosas por hacer); y c) relacionados con el presente ("punta de la lengua", recuerdo de textos).
Fernández-Ballesteros (2009) ha analizado resultados de diversos estudios sobre la ganancia a través del entrenamiento cognitivo en mayores sanos, en comparación con la ejecución en jóvenes. De esta manera confirmó que mejoraban en la realización de tareas cognitivas (velocidad de procesamiento, percepción subjetiva de la memoria cotidiana), aunque la diferencia podría hallarse en la línea base previa al entrenamiento. Calero et al. (2008) diferenciaron la pérdida de memoria asociada al envejecimiento del deterioro patológico en sujetos de entre 60 y 98 años, hallando que no todas las facetas ni tipos de memoria son afectadas por igual con el paso del tiempo. La memoria sensorial, en ausencia de déficit, no sufría efectos y en la memoria a corto plazo no existía declive en ausencia de demencia, excepto en el subsistema de memoria de trabajo.

Otra aportación realizada por Pousada y De la Fuente (2006) evidenciaba las siguientes conclusiones: a) respecto a la capacidad de almacenamiento y velocidad en la pérdida de la información, el ritmo de articulación declinaba con la edad, y se reducía el rendimiento en pruebas de reproducción de estímulos visuales y de reconocimiento; b) en memoria episódica, el reconocimiento de rostros es inferior en mayores; c) en memoria semántica, el nivel de vocabulario en mayores no suele disminuir, sin embargo, los problemas se suelen encontrar en el acceso al léxico, como, por ejemplo, dificultades en la denominación, mayor frecuencia del fenómeno de "la punta de la lengua", o en tareas de fluidez verbal; y d) los problemas en memoria prospectiva acontecen cuando las acciones a recordar están relacionadas con momentos temporales.

\section{Entrenamiento de la memoria: síntesis de programas desarrollados en España}

A continuación se presenta una revisión de diferentes métodos estructurados. En términos generales son programas de carácter multifactorial con un número de sesiones de entre 10 y 16, cuya duración es de 90 minutos, su frecuencia entre 2 y 3 sesiones a la semana y un número de participantes entre 10 y 16 .

\section{Programa de entrenamiento en habilidades de memoria (PEHM) (Fernández-Ballesteros, Izal, Montorio, González y Díaz, 1992)}

Fue creado en la Universidad Autónoma de Madrid y consiste en una serie de módulos, con sesión inicial sobre el funcionamiento de la memoria, posibilidades 
de mejora y falsas percepciones, así como contenidos para ejercitar técnicas (mnemónicos, asociación, pares asociados, visualización, recuerdo de nombres-rostro).

\section{Taller de memoria de Maroto (1999)}

Desarrollado en 12 sesiones, combina ejercicios de técnicas de memoria, así como específicos de estimulación y mantenimiento cognitivo (atención, orientación, fluidez verbal, reminiscencias). Incorpora un cuestionario de quejas subjetivas que permiten su evaluación. Dispone de varios tipos de autoregistros de olvidos para analizarlos a través de explicaciones teóricas, así como cuadernos de tareas de realización en casa.

En relación al terapeuta, añade material teórico e instrucciones para las actividades, además de consejos sobre habilidades en el desarrollo de los talleres (Maroto, 2000).

\section{Método umam de la Unidad de Memoria del Ayuntamiento de Madrid (Montejo, Montenegro, Reinoso, De Andrés y Claver, 2001)}

Consta de 11 sesiones divididas en tres bloques de contenidos: conceptos de memoria, procesos estimulados y aplicaciones a la vida diaria. Se ejecuta en varias fases: a) captación de usuarios y evaluación inicial (cribaje); b) evaluación específica de la memoria y perfil de salud; c) entrenamiento; d) evaluación post-entrenamiento; y e) evaluación final y seguimiento (Montejo-Carrasco, 1998).

Ofrece manuales y cuadernos de ejercicios tanto para el uso de profesionales como usuarios, y material de educación para la salud. Además incorpora un cuaderno de estimulación para el domicilio (Montenegro et al., 2013).

Este programa ha sido analizado a lo largo de sus años de implantación. Los hallazgos en relación con el entrenamiento en 1612 participantes (70,9\% con trastornos de memoria y $66,4 \%$ con pérdida de memoria asociada a la edad) fueron positivos. Mejoró el $37 \%$ del grupo entrenado y alrededor del $80 \%$ de los sujetos con trastornos de memoria. También lograron mejoría en el estado de ánimo y disminución de quejas de memoria (Montejo, Montenegro, Reinoso, De Andrés y Claver, 1999).
Evaluaciones posteriores como la de Montejo (2003) en 1083 usuarios produjo un 40\% de mejoría de la memoria objetiva en el 77\% de los casos, y a nivel subjetivo mejoraron el $75 \%$, porcentaje que incluso llegó a mantenerse durante los seis meses siguientes a su participación.

En otra muestra de 2553 usuarios (Montenegro et al., 2013) el nivel de memoria normal pre-tratamiento fue del $24,8 \%$, y el nivel post del $56 \%$. Sin embargo, cuando realmente existía un trastorno instaurado (débil, moderado y severo) medido a través del test conductual de memoria de Rivermead (RBMT de Wilson, Corkburn y Baddley, 1985) el porcentaje de nivel de rendimiento desciende.

\section{Programa de entrenamiento de la memoria de Puig (2003)}

Se denomina con las siglas PEM y está compuesto por 65 tareas (133 ítems) distribuidas en 36 sesiones, incidiendo en la práctica de técnicas de memoria y entrenamiento de otros procesos cognitivos. Incorpora una técnica de relajación, modificación de creencias negativas y compensación de fallos en las actividades de la vida diaria.

Respecto al procedimiento, se sugiere: a) realizar entrevista preliminar, así como evaluación de screening cognitivo y emocional; b) organización grupal homogénea en edad y funcionamiento cognitivo; c) ensayar cuatro ítems en cada sesión, recogiendo los resultados en un protocolo de respuesta (puntuaciones oscilan entre 0 y 2 puntos, en función de si el ejercicio se ha ejecutado correctamente, máximo 262 puntos), algunos ítems se resuelven de forma individual y otros grupales; y d) incluye un calendario de programación, y encuesta de información subjetiva sobre el programa.

\section{Método UCLM de Hernández-Viadel (2006)}

Diseñado para mayores cuya edad oscila entre 60 y 70 años, los cuales presenten quejas de memoria sin deterioro y buen estado anímico. Compagina sesiones sobre visualización, asociación y recuerdo de nombres, así como ejercicios de estimulación cognitiva, junto con recomendaciones estratégicas para solucionar olvidos cotidianos.

La investigación para evaluar la eficacia del método de la Universidad de Castilla la Mancha, tenía por objetivo reducir las quejas y analizar su efecto en 
la ejecución de tareas de memoria cotidiana y en el estado de ánimo. Fue ensayada con 45 personas, divididas en tres grupos: experimental (recibían 10 sesiones del método); placebo (recibían charlas psicoeducativas); y control (no recibían tratamiento). Valorando resultados con el RBMT, obtuvieron una diferencia de ganancia de 2,7 puntos de media para el experimental al finalizar el entrenamiento, y el control disminuyó 0,51 . En el seguimiento obtuvieron 2,93 y 1,07, respectivamente. En consecuencia, el funcionamiento de memoria de los no entrenados disminuía. Sin embargo, en el experimental se produjo un cambio en la puntuación, de memoria débil a memoria normal, y este cambio se mantuvo e incluso mejoró seis meses después (para una visión más detallada ver Hernández-Viadel, 2006; Latorre-Postigo y Hernández-Viadel, 2008).

\section{Programa Entrenar la memoria e intervenir en reminiscencias de Martínez-Rodríguez (2012)}

Distribuido en dos secciones con directrices para entrenar la memoria y ejercitar reminiscencias. Para memoria incluye 12 sesiones subdivididas en tres bloques: a) explicación teórica; b) estrategias y técnicas (registro información, organización, visualización, asociación, comprensión y retención de textos); c) aplicación del aprendizaje a la vida diaria. Propone realizar la evaluación del rendimiento a través de tareas individuales y la satisfacción de los usuarios.

Estosseis programascomparten diferentes rasgos metodológicos. En la tabla 1 se expone el desglose de contenidos que suelen incorporar sus entrenamientos.

El propósito principal de este artículo es mostrar la efectividad en el entrenamiento de las estrategias de categorización y visualización, la técnica de asociación para el reconocimiento de cara-nombres, así como el método loci, en un grupo de mayores durante su participación en un taller de mejora de la memoria.

\section{Método}

\section{Diseño general}

El taller formó parte de un programa de prevención de la salud física y psíquica implementado por el Servicio Social Comunitario de la localidad de Espera (Cádiz, España) ${ }^{1}$. Su diseño fue multifactorial, modular en 12 sesiones, y compuesto por:

1. Módulo sobre conceptos de memoria, olvidos cotidianos y metamemoria.

2. Entrenamiento de estrategias y técnicas específicas.

3. Ejercicios de mantenimiento cognitivo (atención, percepción y lenguaje) para hacer en casa.

Tabla 1

Revisión de contenidos en programas para el entrenamiento de memoria

\begin{tabular}{|c|c|c|c|c|c|c|}
\hline CONTENIDOS & $\begin{array}{c}\text { UAM } \\
(1992)\end{array}$ & $\begin{array}{c}\text { Maroto } \\
(1999)\end{array}$ & $\begin{array}{l}\text { UMAM } \\
(2001)\end{array}$ & $\begin{array}{l}\text { Puig } \\
(2003)\end{array}$ & $\begin{array}{l}\text { UCLM } \\
(2006)\end{array}$ & $\begin{array}{c}\text { Martínez } \\
\text { (2012) }\end{array}$ \\
\hline Relajación & & & $\checkmark$ & $\checkmark$ & $\checkmark$ & $\checkmark$ \\
\hline Ayudas externas & $\checkmark$ & $\checkmark$ & $\checkmark$ & $\checkmark$ & $\checkmark$ & $\checkmark$ \\
\hline Estimulación cognitiva & & $\checkmark$ & $\checkmark$ & $\checkmark$ & $\checkmark$ & $\checkmark$ \\
\hline \multicolumn{7}{|c|}{ Técnicas codificación y almacenamiento } \\
\hline Método loci & $\checkmark$ & $\checkmark$ & $\checkmark$ & $\checkmark$ & & \\
\hline Asociación & $\checkmark$ & $\checkmark$ & $\checkmark$ & $\checkmark$ & $\checkmark$ & $\checkmark$ \\
\hline Categorización & $\checkmark$ & $\checkmark$ & $\checkmark$ & $\checkmark$ & & $\checkmark$ \\
\hline Visualización & $\checkmark$ & $\checkmark$ & $\checkmark$ & $\checkmark$ & $\checkmark$ & $\checkmark$ \\
\hline Encadenamiento & & $\checkmark$ & & $\checkmark$ & & $\checkmark$ \\
\hline MLP - Reminiscencia & & $\checkmark$ & & & & $\checkmark$ \\
\hline Tareas para casa & & $\checkmark$ & $\checkmark$ & & $\checkmark$ & \\
\hline Aplicación a la vida diaria & & & $\checkmark$ & $\checkmark$ & $\checkmark$ & \\
\hline
\end{tabular}

Nota. Elaboración propia.

1 Para una explicación más detallada, véase Domínguez-Orozco (2011). 


\section{Muestra}

Fueron 21 personas las que formaron el grupo para este taller, aunque solo se presenta la ejecución de tareas en 13 de ellas, debido a varias causas (bajas del grupo, faltas de asistencia y no finalizar las tareas adecuadamente). Los participantes eran todos mujeres, a excepción de un hombre. La media de edad era de 72,84 años (siendo la mínima 63 y la máxima 93 años), y en cuanto al estado civil, el 53,84\% estaban casados, un $30,76 \%$ viudo y el $15,38 \%$ solteros. El nivel de estudios, considerando que el programa estaba contextualizado en un entorno rural, era básico.

\section{Procedimiento}

El taller tenía una duración de 1 hora y 30 minutos con una frecuencia de dos días semanales durante tres meses (de octubre a diciembre del 2006). Fue impartido por una psicóloga, la cual les informó a los participantes en la sesión inicial sobre la metodología, el procedimiento de evaluación, así como de la utilización de los resultados de manera anónima en la evaluación del programa.

El diseño evaluativo del taller fue de intervención media, de un solo grupo con pruebas previas y posteriores, al valorar medidas subjetivas e indicadores de rendimiento de memoria objetiva. El formato de contenido de carácter modular permitió realizar a nivel intrasesional evaluaciones del rendimiento en los ensayos de las cuatro estrategias y técnicas incorporadas al mismo.

El procedimiento metodológico de estas se describe a continuación.

\section{Técnica para el recuerdo de cara-nombre}

Es una aplicación específica de la asociación al recuerdo de nombres y caras. Consiste en realizar una asociación de imagen visual entre rasgos destacables del rostro y el significado del nombre (Montejo, Montenegro y De Andrés, 2004). Su estructuración contempla:

1. Mostrar una plantilla con imágenes de personas y sus nombres asignados.

2. Memorizar mediante repetición.

3. Evaluar rendimiento pre-test.

4. Explicación técnica: estudiar y fijar rasgos característicos (modo de vestir, rasgos físicos faciales, buscar similitudes con nombres de personajes populares o de su entorno) a fin de asociarlo con su nombre.

5. Realizar pos-test.

\section{Estrategia de categorización}

Se describe como una estrategia de organización de la información. Se trata de realizar agrupaciones por categorías del material a recordar, usualmente un listado de elementos, palabras (como una lista de compras), etc. Se optimiza la retención de las categorías principales que evocan los elementos incluidos en ellas. Recordar ítems mediante las unidades semánticas de los atributos que comparten facilita la recuperación posterior de la información (Gross et al., 2012).

\section{Estrategia de visualización}

Consiste en la fijación de imágenes visuales y la utilización de referencias visuoespaciales sencillas para la facilitación del recuerdo. Aquí se ha presentado el ejercicio denominado "Sonrisas" (Puig, 2003). Consiste en:

1. Presentar una lámina con una distribución de 18 círculos, nueve de ellos con el dibujo de una sonrisa, y localizaciones a memorizar.

2. Explicación técnica: elaborar y crear la imagen mental de la lámina con claves espaciales sencillas.

3. Retirar lámina tras un minuto.

4. Presentar una plantilla con círculos en blanco, en la cual se localiza la posición de las sonrisas.

Es una estrategia simple de entrenamiento de memoria sensorial visual y a corto plazo para el registro de la información, incidiendo en el apoyo en referencias espaciales básicas y recorridos mentales de imágenes.

\section{Método loci}

Se trata de establecer una serie de ubicaciones en un espacio perfectamente conocido para el sujeto (su casa, un trayecto que realice habitualmente, etc.), y asociar a cada una de ellas, de forma ordenada, la imagen de cada una de las acciones a aprender. Útil para recordar una lista de tareas o una lista de objetos, siempre que sea importante el orden de los elementos en la lista. Trabaja la memoria prospectiva mediante la imaginación, asociación de acciones y la referencia espacial correspondiente (locis). 
Comprende el siguiente desarrollo:

1. Presentación de la lista de acciones a recordar.

2. Asociar cada acción con un lugar (puede utilizar como material de apoyo el plano de una casa). Por cada estancia "fijar mentalmente" una imagen de los ítems de la lista de tareas a realizar.

3. Posteriormente, en las estancias consecutivas seguir asociando una acción de la lista de cosas en el orden de listado. Ejemplo: Primera acción, comprar un alimento ("colocarlo" en el felpudo de la entrada de la casa); segunda acción, ir al médico, fijar la imagen en el recibidor; y así sucesivamente.

4. Al finalizar, repasar el plano y reparar en los objetos de cada estancia asociado a cada acción, de manera que se facilite el recuerdo objeto/acción y el orden de realización (Maroto, 1999; Montejo y Montenegro, 2006).

A continuación se presenta el análisis del rendimiento en los ejercicios realizados de estos cuatro procedimientos mnemotécnicos.

\section{Resultados}

\section{Asociación cara-nombre}

Se realizaron tres ensayos de entrenamiento de esta técnica con diferentes imágenes de personas. Para el ensayo 1 y 3 se utilizaron láminas que contenían 12 rostros con sus respectivos nombres. El ensayo 2 incluía siete imágenes de rostros con los nombres y dos diapositivas intercaladas sin imagen, aunque con un nombre propio designado.

Con el fin de explorar el rendimiento logrado, en primer lugar se obtuvieron el número de aciertos por cada participante, cuyas puntuaciones directas fueron tipificadas y analizadas a través del coeficiente de correlación de Pearson ( $\rho$ xy). Una vez obtenido el significado correlacional mediante la tabla de Guilford, se procedió a calcular la prueba de significación $\tau$ de Student-Fisher (para muestras pequeñas $n<30$ ) por cada ensayo de entrenamiento (E) (ver tabla 2).

Respecto a la correlación de Pearson, los ensayos 1 y 3 mostraron una correlación positiva alta $(0,60<$ $\rho>0,80)$, y el E2 una correlación media $(0,40<\rho>$ $0,60)$. Al comprobar la significación de este coeficiente, el E1 resultó significativo en tres niveles de significación y 10 grados de libertad (gl.) $(\tau>$ a $0,05=1,812$; a $0 ., 01=2,764 ; \alpha 0,05=3,169)$. El E3 también manifestó significación estadística positiva en los tres niveles de significación para 8 gl. ( $\tau>$ a $0,05=1,860$; $\alpha$ $0,01=2,896 ; \alpha 0,005=3,355)$. Esta conclusión estadística indicaba la ganancia en el recuerdo tras el entrenamiento. Sin embargo, el E2 no resultó significativo $(\tau<\alpha 0,05$ y $9 \mathrm{gl}=1,833)$, la técnica no tuvo efecto de mejora en el recuerdo para esta tarea.

\section{Categorización}

En la figura 1 se expresa el resultado descriptivo correspondiente a la práctica de la categorización mediante el ejercicio "Lista de palabras". Se analizó mediante el recuento del número de palabras recordadas en dos ensayos diferenciados temporalmente, durante la sesión de intervención.

Los participantes tenían que memorizar una lista de 20 palabras a través de la repetición simple (E1, recuerdo "sin técnica"), y posteriormente, tras la explicación teórica de la estrategia, se ensayaban las agrupaciones para su posterior recuerdo (E2, aciertos "con técnica"). La lista de palabras se clasificaba en cuatro categorías: colores, frutas, profesiones y utensilios de cocina.

Tabla 2

Estadísticos descriptivos y de correlación para tres ensayos de la técnica rostros y nombres

\begin{tabular}{ccccccc}
\hline & \multicolumn{2}{c}{ ENSAYO $\mathbf{1}(\mathbf{n}=\mathbf{1 2})$} & \multicolumn{2}{c}{ ENSAYO 2 $(\mathbf{n}=\mathbf{1 1})$} & \multicolumn{2}{c}{ ENSAYO 3 $(\mathbf{n}=\mathbf{1 0})$} \\
\hline & Medidas pre & Medidas post & Medidas pre & Medidas post & Medidas pre & Medidas post \\
\hline$\mu$ & 4 & 7,75 & 3,9091 & 7 & 5,8 & 8,1 \\
$\delta$ & 2,0412 & 2,2032 & 1,7296 & 2,1320 & 2,7856 & 4,0112 \\
$\rho$ & & & & 0,4523 & 0,7786 \\
$\tau$ & & & & 1,5214 & $3,5093^{* * *}$ \\
\hline
\end{tabular}

Nota: ${ }^{* * *}$ Niveles de significación. Elaboración propia. 
El entrenamiento de la técnica obtuvo un rendimiento de recuerdo superior en todos los participantes respecto al recuento de palabras recordadas inicial (ejemplo: para el caso N. $7 \mathrm{E} 1=7$ palabras/E2 $=19$ palabras). La diferencia de medidas total en el número de palabras recordadas obtenida en ambos ensayos fue de 8,3 palabras tras el ensayo "con técnica".

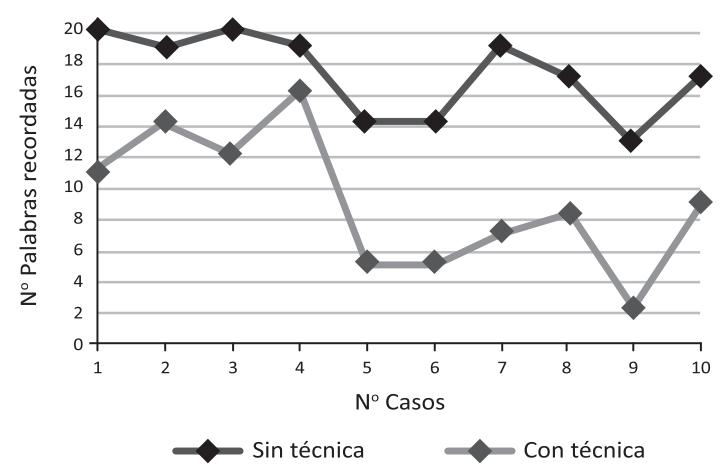

Figura 1. Resultados desarrollo de la técnica de categorización. Elaboración propia.

\section{Visualización}

En el gráfico 2 se exponen datos del ejercicio realizado en dos momentos temporales, delimitando aciertos y confusiones en la ubicación de las localizaciones de sonrisas. Se consideraron confusiones los errores de localización y las omisiones de las mismas. En global, el número de aciertos tuvieron una media pos-test de 7,2 del total de nueve localizaciones (ejemplo: para el caso N. 8 Epretest = obtiene 4 localizaciones correctas y 5 confusiones; Epostest $=$ señala las 9 localizaciones en la plantilla aunque añade dos confusiones).

Los participantes que habían finalizado el entrenamiento y señalado menos confusiones realizaron una mejor representación espacial y consecuentemente, habían elaborado mejores coordenadas espaciales para la localización posterior de las sonrisas, obteniendo mayor registro visual de la imagen.

\section{Método loci}

Como se puede comprobar visualmente en la figura 3, los hallazgos de la valoración realizada en el pos-test respecto al número de aciertos de la lista de acciones a recordar, en líneas generales, es superior tras la exposición y ensayo del método. La corrección del ejercicio consideraba dos puntuaciones: el acierto

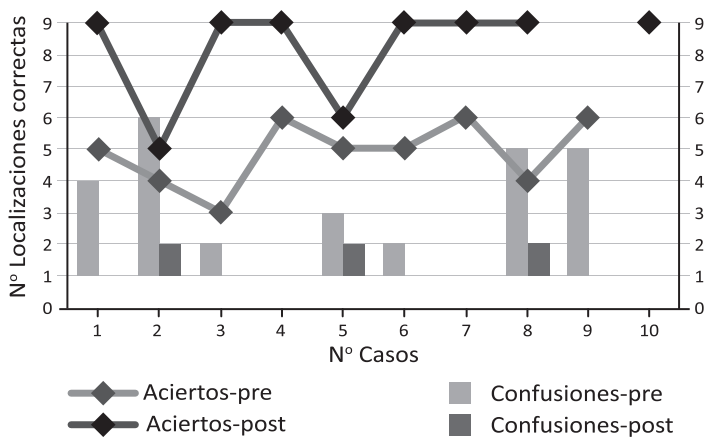

Figura 2. Resultados técnica visualización: Ejercicio Sonrisas. Elaboración propia.

de la acción concreta ( 0,5 puntos), y la ubicación en el orden inicial (valoración de 1 punto, máximo 10 puntos). La diferencia de las medias global en el recuerdo de acciones fue de 2,25 número de acciones más en el ensayo pos-test.

En cuanto a la ubicación del orden, se observó la tendencia general de los efectos de primacía y recencia, pues desde los ítems 4 a 8 se producían mayores confusiones de orden. Para aclarar la corrección de la prueba, se explicó un ejemplo del ejercicio: el caso N. 1 logró recordar todas las acciones en el orden propuesto; había obtenido 6,5 puntos en Epre-test (3 primeras y 2 últimas acciones en orden correcto $=5$ puntos +3 acciones en orden alterado $=1,5$ puntos). La ganancia en el recuerdo tras la asimilación del método fue de 3,5 acciones más.

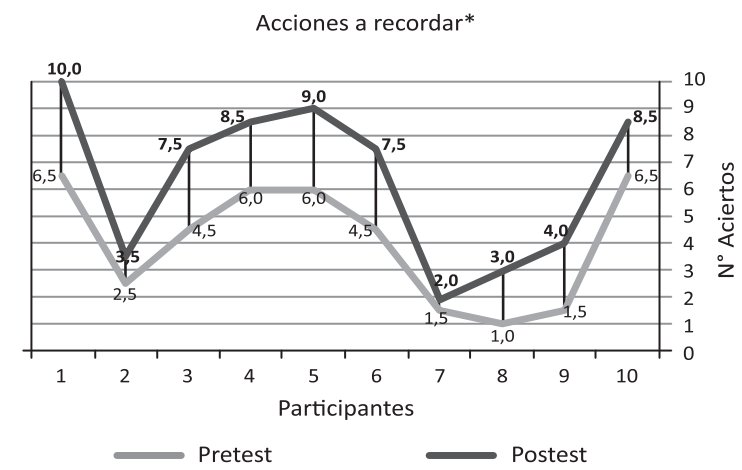

Figura 3. Comparación pre y pos-test de los resultados en método loci. Relación de acciones: 1 . Comprar zanahorias y puerros para el puré; 2. Comprar un cuaderno a mi nieta; 3 . Ir al médico a pedir la receta de la pastilla de la tensión; 4 . Comprar una bufanda marrón; 5. Comprar comida para el canario; 6. Comprar el periódico para mi marido; 7. Pagar el recibo de la luz; 8. Comprar un bote de colonia; 9. Comprar el pan integral en la panadería; 10. Ir a la ferretería a por puntillas. Elaboración propia. 


\section{Discusión general}

Una cuestión clave sobre eficacia en entrenamiento de memoria es la elección de estrategias y técnicas a emplear, las cuales permitan conseguir resultados más óptimos en el diseño de un programa (MontejoCarrasco, 1998). Un metaanálisis realizado por Gross et al. (2012) evidenciaba esta relación. Los estudios seleccionados en este clasificaban las estrategias mnemotécnicas en dos tipos: comunes (repetición, asociación, categorización, imaginación y concentración), y combinadas (reconocimiento cara-nombre, mnemotecnia para números e historias y el método loci). Así mismo, indicaba que el total de números de estrategias estaba asociado positivamente con el incremento en la diferencia pre y post-entrenamiento. La mayoría solía enseñar - entre dos y siete diferentes fue la combinación más común-al menos tres de las siguientes: método loci, asociación, categorización y visualización. La más prevalente era la visualización, seguida de la técnica de recuerdo cara-nombre.

En otro metaanálisis, Wilson (2005) integró diferentes investigaciones sobre la efectividad de programas de entrenamiento en mejorar la memoria subjetiva en mayores. Valorando la magnitud de los tamaños de efecto en rendimiento de memoria, reveló, a través de un modelo de regresión, qué predictores eran significativos para altos tamaños, los cuales fueron las intervenciones multifactoriales y el uso de nuevas tecnologías.

El diseño de este entrenamiento evidenció efectos positivos en el rendimiento del recuerdo. La aplicación de las estrategias de visualización y categorización, la técnica de asociación cara-nombre y el método loci, generaron resultados de efectividad.

Existe poca evidencia empírica con respecto al uso de estrategias mnemónicas y técnicas específicas para mejorar la memoria, y dotar de efectividad al entrenamiento. Gross y Rebok (2011) describieron el uso de estrategias mnemotécnicas de clasificación (subjetiva, serial y semántica) en el aprendizaje de listas de palabras, y su relación con el rendimiento de memoria, así como la asociación con el funcionamiento diario y sus efectos a largo plazo, en una muestra de participantes del estudio ACTIVE (Advanced Cognitive Training for Independent and Vital Elderly o Entrenamiento Cognitivo Avanzado para Mayores Vitales e Independientes). Encontraron que variables sociodemográficas tales como ser mayor, más joven, mujer, tener mejor salud y con más años de nivel educativo, influía en la utilización de estrategias de clasificación subjetiva y/o semántica (uso de categorizaciones por significado relacional) en el aprendizaje de listas de palabras. Y si esas listas no tenían conexiones, usaban una estrategia de tipo subjetiva y/o serial (recuerdo de ítems de manera consecutiva). Sus análisis revelaron que a través del entrenamiento, el nivel de uso de estrategia mejoraba, y se mantuvo en la evaluación de seguimiento en los cinco años posteriores (si bien la edad no modificó esta asociación).

En el estudio realizado por la autora de este artículo la mayoría de las participantes compartían esas características sociodemográficas (76,9\% mujeres entre 65 y 75 años, y solo un hombre), de manera que a fin de valorar la tarea de recuerdo de listas de palabras, se tomó como indicador del patrón de rendimiento las puntuaciones medias del número total de palabras (medidas pre y pos-test), obteniendo mayor rendimiento después de haber realizado el aprendizaje de la estrategia de categorización. Ciertamente exhibir resultados superiores post-hoc implicaba la obtención de mejores niveles de codificación.

Similar a la conclusión antes comentada (Gross y Rebok, 2011), la organización semántica de la información había incrementado la retención y accesibilidad al recuerdo, lo cual cobra importancia debido a su implicación en el quehacer diario para personas mayores, como, por ejemplo, recordar los productos de la compra.

Respecto a la estrategia de visualización, la comparación de ensayos resultó igualmente con incremento positivo. El uso de esta estrategia maximizó el proceso atencional y la fijación en el sistema de memoria sensorial visual. Involucrar estrategias de imaginación visual en los entrenamientos produce beneficios en los mayores debido a que la región occipital del cerebro, responsable del procesamiento de información visual, suele conservarse durante más tiempo en ausencia de lesiones.

Con relación a lo anterior, McDougall (2000) mejoró competencias en memoria prospectiva como "pedir una cita, buscar pertenencias o comunicar un mensaje a alguien", a mayores participantes en el programa Memorias, memorias: ¿puede mejorarse?, cuyos contenidos contemplaba el uso de técnicas tales como la organización (categorización), y habilidades de imaginación visual (visualización).

El método loci es otra técnica mnemotécnica que cuenta con visualización de manera combinada (Wilson, 2005). Facilita y favorece las condiciones idóneas para recuerdos de carácter episódico (mediante el uso de asociaciones con localizaciones espaciales), las cuales consecuentemente son dependientes 
del contexto, de tal forma que este método al basarse en el principio de codificación específica y proporcionarse el soporte ambiental, optimiza los resultados (Fernández-Ballesteros, Moya, Iñiguez y Zamarrón, 1999). Las conclusiones obtenidas en el ensayo mostraron ganancias efectivas en el recuerdo de las acciones a recordar. El contenido de la tarea a entrenar involucraba la memoria prospectiva. Recordar "hacer cosas" (acordarse de llevar a cabo una acción en el futuro) es importante para que los mayores mantengan la vida independiente, es memoria de eventos que han sido planificados pero no han ocurrido (componente retrospectivo, "acordarse de la acción que hay que llevar a cabo"). La aplicación del método incluía además recordarlas en el orden de presentación correcto, $y$ fue en esta fase en la que los participantes mostraron más errores. De acuerdo con Gross y Rebok (2011) asumir el orden de recuerdo reflejaba el proceso de almacenaje, lo cual está asociado a la memoria de trabajo que suele declinar con la edad.

Estos logros son congruentes con los que pusieron de manifiesto Cavallini, Pagnin y Vecchi (2003) sobre el efecto del método loci en el rendimiento de memoria utilizando tareas ecológicas. Sin embargo, estos autores señalaron que el efecto fue diferente si se evaluaba la habilidad de reutilización de la técnica en tareas no familiares.

En el caso de la técnica de asociación cara-nombre, se produjo significación positiva en el ensayo inicial y en el posterior, el cual había sido distanciado temporalmente (espaciamiento por sesiones). Como sugirieron McDaniel y Bugg (2012), la retención de estrategias adquiridas se facilitó al espaciar los entrenamientos. Esto es aplicable a los resultados de este ensayo puesto que si se valoran las puntuaciones de los sujetos en el pre-entrenamiento de ensayos 1 y 3 , cinco de ellos tenían mayor cantidad de recuerdo en el E3 y la diferencia de ganancia fue de 3,8 palabras más, indicativo del uso de la estrategia de clustering, aprendida en la sesión de entrenamiento anterior. Sin embargo, el E2 no resultó válido, pese a que contenía un menor número de ítems y se ejecutó seguidamente al E1. Pensamos que se debe al propio contenido más complejo de la tarea, es decir, el diseño de este ensayo se distinguía en que se debía recordar dos nombres sin que la imagen de la cara estuviera presente (posiciones 7 y 8 de 9 diapositivas), y las tareas de reconocimiento son más sencillas que el recuerdo (recuerdo pre-test para estos dos ítems fue un (1) acierto y en ubicación desacertada, frente a 14 aciertos del total en el pos-test).
El recuerdo de caras es complejo y compromete a la memoria episódica, la cual involucra el proceso obligatorio de realizar asociaciones entre el estímulo, la imagen de la persona concreta, y cómo se denomina el estímulo (el nombre concreto). El envejecimiento ocasiona déficits asociativos, lo cual atañe al funcionamiento disminuido de zonas cerebrales como el hipocampo y la corteza prefrontal (Old y Naveh-Benjamin, 2008, citado en Small, Rawson, Eisel y McEvoy (2012). Igualmente, intervienen otras regiones cerebrales tales como la corteza visual occipital para el acceso e interpretación de las imágenes, área frontotemporal para la organización e identificación de rasgos, hipocampo para acceder a los recuerdos relacionados con las personas, y polos temporales para acceder al propio nombre (Montejo y Montenegro, 2006).

Un evento cotidiano específico vinculado al recuerdo de nombres de personas, y el cual constituye una queja subjetiva de memoria, es el fenómeno de la punta de la lengua (PDL). En este sentido JuncosRabadán, Facal, Álvarez y Rodríguez (2006) analizaron la producción y resolución de PDL concluyendo que existía un efecto significativo de la edad en relación con la producción de mayor número de PDL en nombres propios de personas, y menor en nombres propios de lugares (déficits evidentes a partir de los 70 años), así como la resolución se producía más favorablemente a través de primado fonológico y no evocación libre. Todas estas implicaciones son interesantes y deberían ser tenidas en cuenta en el diseño de los objetivos del entrenamiento y para mejorar el desarrollo de las técnicas.

La medición de la eficacia del diseño general sirve como acreditación de los resultados de efectividad de las estrategias y técnicas aplicadas. La evaluación sumativa produjo una disminución de quejas subjetivas valoradas a través del cuestionario de Maroto (1999). Por ejemplo, quejas relacionadas con la memoria prospectiva como "ir a la compra y olvidar algún producto" obtuvo una diferencia pos-test de - 0,61 ; o en el ítem "no recodar el nombre de personas conocidas", la diferencia $\mu$ grupal fue de $-0,20$, indicando el decremento de puntuaciones tras el entrenamiento completo. Por tanto, los logros fueron coherentes y congruentes respecto a la evaluación general del entrenamiento (Domínguez-Orozco, 2012), si bien es necesario realizar más investigación orientada a valorar el impacto de entrenamiento de estrategias y técnicas, tanto a largo plazo, como en lo relativo a la trasferencia en rendimiento de memoria a la vida cotidiana. 
Cabe añadir que algunas características de efectividad de intervenciones cognitivas favorecen la prevención del deterioro de memoria asociado a la edad. Novoa, Juárez y Nebot (2008) determinaron qué intervenciones eran más efectivas y qué efectos generaban (metaanálisis de estudios desde 1987 al 2007). Describieron que el entrenamiento cognitivo aumentaba el rendimiento de la memoria reciente verbal y de asociación, que existía poca evidencia respecto a la memoria inmediata, remota y memoria subjetiva, y por último, no hallaron estudios sobre memoria sensorial, ni demostración de transferencia del material entrenado a otros dominios específicos.

Esto tiene relación con la hipótesis que encontramos en la literatura sobre cómo el uso de estrategias de memoria asiste a componentes cognitivos del funcionamiento diario, subyacentes en actividades básicas e instrumentales de la vida diaria, lo cual debería ser considerado en futuras líneas de investigación.

Este estudio adolece de limitaciones pues la muestra de participantes es pequeña y, con el fin de robustecer estos resultados, podría incorporarse un grupo de control comparativo (sin ensayos) al medir el rendimiento en estrategias, así como analizar el efecto longitudinal de los resultados e incluir una medida objetiva de memoria, cuestiones que claramente son sugerencias para otros estudios.

Por último, es necesario aclarar que otras causas de alteraciones de memoria tales como creencias de autoeficacia sobre rendimiento percibido de memoria, déficit motivacionales o afectivos tienen su influencia, y son también variables a tener en cuenta en el diseño y valoración de la eficacia de los programas (Fernández-Ballesteros, et al., 1999).

\section{Referencias}

Cavallini, E., Pagnin, A. y Vecchi, T. (2003). Aging and everyday memory: The beneficial effect of memory training. Archives of Gerontology and Geriatrics, 37, 241-257. doi: 10.1016/S0167-4943(03)00063-3

Calero, M. D. (2000). Psicología de la vejez: funcionamiento cognitivo. En R. Fernández-Ballesteros, (Dir.). Gerontología Social (pp. 201-223). Madrid: Pirámide.

Calero, M. D., Navarro, E., Gómez, L., López, A., Torres, I. y Calero, M. J. (2008). Olvidos y memoria: relaciones entre memoria objetiva y subjetiva en la vejez. Revista Española Geriatría y Gerontología, 43(5), 299-307. doi: 10.1016/S0211-139X (08)73572-8
Calero-García, M. D. y Navarro-González, E. (2006). Eficacia de un programa de entrenamiento en memoria en el mantenimiento cognitivo de ancianos con y sin deterioro cognitivo. Revista de Psicología de Clínica y Salud, 17(2), 187-202. Recuperado de: http:// www.copmadrid.org/webcopm/resource.do?recurso $=4000 /$ \&numero $=2006172$

Cortés, J. A. y Estrada, I. (2000). Entrenamiento cognitivo: Taller de memoria en tercera edad. Información Psicológica, 74, 66-71.

Domínguez-Orozco, M. E. (2011). La intervención con mayores desde los servicios sociales comunitarios: programas de promoción de la salud. Revista Electrónica Tiempo, Portal de la Psicogerontología, 27. Recuperado de www.psicomundo.com/tiempo/tiempo27.htm

Domínguez-Orozco, M. E. (2012). Olvidos cotidianos en personas mayores. Revista Electrónica de Psicología Iztacala, 15(4), 1294-1309. Recuperado de http://www. revistas.unam.mx/index.php/repi/article/ view/34738

Fernández-Ballesteros, R. (2009). Envejecimiento activo: Contribuciones desde la Psicología. Madrid: Ediciones Pirámide.

Fernández-Ballesteros, R., Moya, R., Iñiguez, J. y Zamarrón, M. D. (1999). ¿Qué es la psicología de la vejez? Madrid: Biblioteca nueva.

Fernández Ballesteros, R., Izal, M., Montorio, I., González, J. L. y Díaz, P. (1992). Evaluación e intervención psicológica en la vejez. Barcelona: Ed. Martínez Roca.

Gross, A. L., Parisi, J. M., Spira, A. P., Kueider, A. M., Ko, J. Y. y Saczynski, J. S. (2012). Memory training interventions for older adults: A meta-analysis. Aging and Mental Health, 16(6), 722-734. doi: 10.1080/13607863. 2012.667783.

Gross, A. L. y Rebok, G.W. (2011). Memory training and strategy use in older adults: Results from the ACTIVE study. Psychology and Aging, 26(3), 503-517. doi: 10.1037/a0022687.

Hernández-Viadel, J. V. (2006). Método U.C.L.M. para el Entrenamiento de memoria en personas mayores. (Tesis inédita de doctorado, Cuenca, Ediciones de la Universidad de Castilla la Mancha. Recuperado de https://ruidera.uclm.es/xmlui/bitstream/handle/10578/ 950/217\%20M\%C3\%A9todo\%20UCLM.pdf?sequen$\mathrm{ce}=1$

Juncos-Rabadán, O., Facal, D., Álvarez, M. y Rodríguez, M. S. (2006). El fenómeno de la punta de la lengua en el proceso de envejecimiento. Psicothema, 18(3), 501506. Recuperado de: http:// www.psicothema.com/ pdf/3244.pdf 
Latorre Postigo, J. M. y Hernández Viadel, J. V. (2008). Método UCLM de entrenamiento de la memoria en mayores. Cuenca: Ediciones de la Universidad de Castilla-La Mancha.

McDaniel, M. A. y Bugg, J. M. (2012). Memory training interventions: What has been forgotten? Journal of Applied Research in Memory and Cognition, 1(1), 45-50. doi: 10.1016/j.jarmac.2011.11.002

McDougall, G. J. (2000). Memory improvement in Assisted Living Elders. Issues Mental Health Nursing, 21(2), 217-233. Recuperado de: http://www.ncbi.nlm.nih. gov/pmc/articles/PMC2519137/pdf/nihms61377.pdf

Martínez-Rodríguez, T. (2012). Entrenar la memoria e intervenir en reminiscencias. Madrid: Editorial Médica Panamericana.

Maroto, M. A. (1999) Taller de memoria (Estimulación y mantenimiento cognitivo en personas mayores). Madrid: TEA.

Maroto, M. A. (2000). La memoria: programa de estimulación y mantenimiento cognitivo. Madrid: Instituto de Salud Pública. Recuperado de http://www.imsersomayores.csic.es/documentos/documentos/maroto-memoria-01.pdf

Montenegro, M., Montejo, P., Claver-Martín, M. D., Reinoso, A.I., De Andrés-Montes, M. E., García-Marín, A., ... Huertas, E. (2013). Relación de las quejas de memoria con el rendimiento de memoria, el estado de ánimo y variables sociodemográficas en adultos jóvenes. Revista Neurología, 57(9), 396-404. Recuperado de: http://www.revneurol.com/sec/resumen.php?or= pubmed\&id=2013047

Montenegro, M., Montejo, P., Reinoso, A. I., De Andrés, M. E. y Claver, M. D. (2013). El método UMAM de entrenamiento de Memoria. Centro de Prevención del Deterioro Cognitivo. Madrid: Ayuntamiento de Madrid.

Montejo, P. y Montenegro, M. (2006). Memoria cotidiana en los mayores. Portal Mayores, Informe Portal Mayores, N. ${ }^{\circ}$ 60. Lecciones de Gerontología, VIII. Recuperado de http://www.imsersomayores.csic.es/documentos/ documentos/montejo-memoria-01.pdf

Montejo, P., Montenegro, M. y De Andrés, M. E. (2004). Mejora y mantenimiento de la memoria en los mayores. En S. Ballesteros-Jiménez (Coord.), Gerontología. Un saber multidisciplinar (pp. 546-594). Madrid: Editorial Universitas.

Montejo, P. (2003). Programa de entrenamiento de memoria para mayores con alteraciones de memoria: resultados y predictores. Revista Española de Geriatría y Geron- tología, 38(6), 316-326. Recuperado de: http://www. elsevier.es/es-revista-revista-espanola-geriatria-gerontologia-124-articulo-programa-entrenamiento-memoria-mayores-con-13056961

Montejo, P., Montenegro, M., Reinoso, A. I., De Andrés, M. E. y Claver M. D. (2001), Programa de Memoria, Método UMAM. Madrid: Ayuntamiento de Madrid. Área de Salud y Consumo.

Montejo, P., Montenegro, M., Reinoso, A. I. De Andrés, M. E. y Claver, M. D. (1999). Estudio de la eficacia de un programa de entrenamiento de memoria multicéntrico para mayores de 60 años. Revista Española de Geriatría y Gerontología, 34(4), 199-208.

Montejo-Carrasco, P. (1998). Programas de memoria. En Actas del $V$ Congreso Estatal de Intervención Social: Calidad y responsabilidad compartida: retos del bienestar en el cambio de siglo. Madrid: Ministerio de Trabajo y Asuntos Sociales.

Novoa, A. M., Juárez, O. y Nebot, M. (2008). Efectividad de las intervenciones cognitivas en la prevención del deterioro de la memoria en las personas mayores sanas. Gaceta Sanitaria; 22(5), 474-482. Recuperado de: http://scielo.isciii.es/pdf/gs/v22n5/revision.pdf

Pousada, M. y De la Fuente, J. (2006). Memoria y atención. En Triadó, C. y Villar, F. (Coords.), Psicología de la vejez (pp.113-139). Madrid: Alianza Editorial.

Puig, A. (2003). Programa de entrenamiento de la memoria. Madrid: Editorial ccs.

Small, B. J., Rawson, K. S., Eisel, S. y McEvoy, C. L. (2012). Memory and Aging. En Krauss, S. y Sliwinski, M. (Eds.), The Wiley-Blackwell Handbook of Adulthood and Aging (pp. 174-189). Blackwel Publishing: UK

Stern, Y. (2002). What is cognitive reserve? Theory and research application of the reserve concept. Journal of the International Neuropsychological Society, 8(3), 448460. doi: 10.1017/S1355617702813248

Stern, Y. (2013). Cognitive Reserve: Implications for assessment and intervention. Folia Phoniatrica et Logopaedica, 65(2), 49-54. doi: 10.1159/000353443

Wilson, B. A., Cockburn, J. y Baddeley, A. (1985). The Rivermead Behavioural Memory Test. Titchfield, UK: Thames Valley Test Company.

Wilson, K. Y. (2005). The Effectiveness of Memory Training Programs in improving the subjective memory characteristics of Healthy Older Adults with Memory Complaints: a Meta-Analysis. Theses, Dissertations and Capstones. Paper 165. Recuperado de: http://mds. marshall.edu/etd/165 